\title{
2-year survival among elderly hospitalised for acute respiratory infection versus hip fracture: a useful comparison to raise awareness
}

\author{
To the Editor:
}

We read with great interest the article by CillónIz et al. [1]. The authors nicely reported and discussed recent literature showing that pneumonia is a common lung infection that can be life-threatening, with particular concern for the elderly. Indeed, the annual incidences of hospitalisation for respiratory infections increase with age from $0.2 \%$ (for patients aged $<75$ years) to $1.9 \%$ (for age $80-84$ years), $3.2 \%$ (for age $85-89$ years) and $5.0 \%$ (for age $\geqslant 90$ years) [2]. For the elderly, pneumonia has the greatest risk of death among the common causes of hospitalisation [3]. We do agree with the authors when they concluded that preventive interventions are of pivotal importance to improve outcomes and reduce the occurrence of adverse consequences [1]. However, we would like to emphasise that there is a mismatch between the high morbidity and mortality caused by respiratory infection and the low public awareness of this disease. A large pneumonia awareness survey involving over 9000 adults aged $\geqslant 50$ years highlighted that most fail to accurately gauge their own pneumonia risk, leading to inadequate pneumonia prevention efforts including low uptake of existing vaccines [4]. The low public awareness of respiratory infection risk and severity in the elderly is a barrier to healthcare delivery and a driver of unhealthy ageing [5]. It is critical to raise awareness of this disease among the general public to improve the management of this largely preventable infectious disease [5].

On the contrary, breaking a hip is widely recognised as a major concern for the elderly [6]. Breaking a hip or pneumonia do not share pathophysiologic features, but they are both acute events with important long-term consequences. Consequently, hip fracture might serve as a useful comparator for highlighting the life-threatening consequences of acute respiratory infection in the elderly. Our subjective impression is that the news of an elderly relative breaking a hip tends to raise more concern from the family than news of a pneumonia diagnosis. The objective of the study was to compare 2-year outcomes for elderly (aged $\geqslant 80$ years) patients after hospitalisation for acute respiratory infection or hip fracture.

A 2009-2015 population-based cohort study was performed in the Centre-Val de Loire region of France (2.5 million inhabitants), using medico-administrative data collected from the national hospital discharge databases. We defined cases of patients aged $\geqslant 80$ years who were hospitalised for at least one night for acute respiratory infection or hip fracture using ICD-10 and current procedural terminology algorithms $[2,7]$. For each patient, the following data were extracted: age, sex, primary diagnosis, comorbidities and hospital frailty risk at admission (according to $[8,9]$ ). The reuse of already recorded and anonymised data falls within the scope of the French Reference Methodology MR-005 (2016-41 law) which require neither information nor non-opposition of the included individuals. Access to linked anonymous file in the PMSI databases was approved by the French National Commission for Data Protection and Liberties (CNIL MR-005 number 4116221019). The main outcome was the 2 -year mortality of the two patient groups, referring to the mortality in hospital, which was defined as death during one readmission over the 2-year follow-up. We used KaplanMeier estimates to describe the overall survival. Cox proportional hazards models were used to determine the effect of the acute respiratory infection, as well as time-period, on the risk of death. Hazard ratios and 95\%

@ERSpublications

If breaking a hip feels like a concern for the elderly, then getting pneumonia should be twice as concerning: patients hospitalised for lung infection had 3.3-fold greater in-hospital mortality and 1.8fold increased risk of death at 2 years https://bit.ly/2Xqsrf6

Cite this article as: Guillon A, Mizgerd JP, Grammatico-Guillon L. 2-year survival among elderly hospitalised for acute respiratory infection versus hip fracture: a useful comparison to raise awareness. Eur Respir Rev 2020; 29: 200156 [https://doi.org/10.1183/16000617.0156-2020]. 
FIGURE 1 Kaplan-Meier curves showing the cumulative probabilities of survival at 2 years for elderly patients discharged from hospital after acute respiratory infection or hip fracture (log rank test $p<0.0001)$.

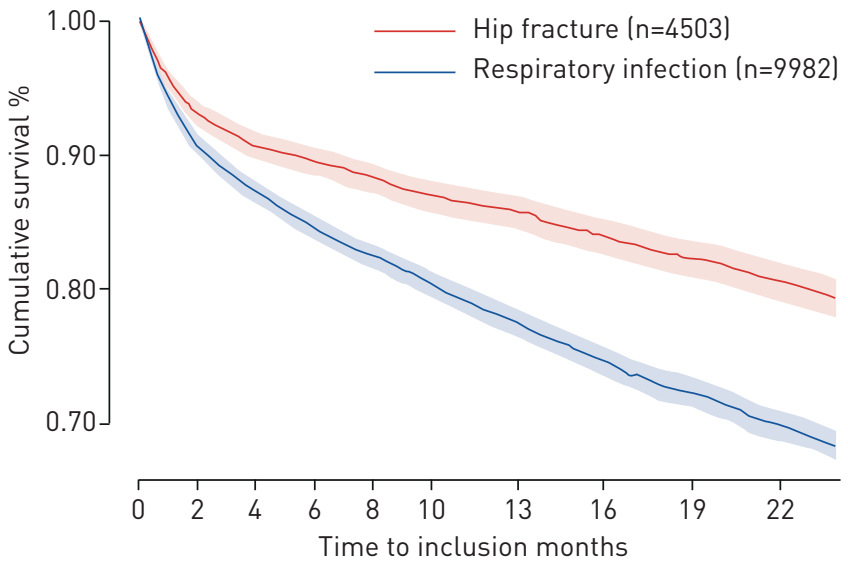

confidence intervals were calculated. Proportionality of hazards and log-rank test were calculated using SAS, and statistical analyses were carried out using R software [10].

We included 16917 patients aged $\geqslant 80$ years hospitalised for acute respiratory infection $(n=12159)$ or hip fracture $(n=4758)$. Patients hospitalised for acute respiratory infection had more comorbidities and more elevated Frailty Score compared to patients hospitalised for hip fracture: cancer: $12 \%$ versus $6.5 \%$; chronic heart diseases: $62.6 \%$ versus $28.3 \%$; chronic pulmonary diseases: $19 \%$ versus $3 \%$; chronic renal diseases: $11 \%$ versus $5.4 \%$; diabetes: $15 \%$ versus $8.9 \%$; neurological diseases: $32.3 \%$ versus 35.2 ; obesity: $6.3 \%$ versus 1.7\%; Frailty Score: $5.5 \pm 6.9$ versus $3.9 \pm 6.1$ for acute respiratory infection versus hip fracture, respectively. The in-hospital mortality during the stay was 3.3-fold greater for patients hospitalised for acute respiratory infection (17.9\% mortality for respiratory infection and 5.4\% for hip fracture). The Kaplan-Meier curves for elderly patients discharged from hospital after acute respiratory infection $(n=9982)$ or hip fracture $(n=4503)$ showed a significant difference in 2-year mortality between the two groups (log rank test $\mathrm{p}<000.1$ ) (figure 1). Importantly, even after adjusting for the confounding factors of comorbid conditions and Frailty Score (as well as age and sex), the global risk of death at 2 years for elderly patients hospitalised for respiratory infection compared to the hip fracture was significantly elevated (HR 1.8, 95\% CI 1.7-2.0). If breaking a hip feels like a concern for the elderly, then getting pneumonia should be twice as concerning.

Our study had limitations. The use of administrative hospital databases introduced an inherent bias that should be taken into consideration. The strengths and limitations of using healthcare databases for epidemiological purposes have already been extensively discussed [2, 11, 12]. Healthcare provided for elderly patients varies across diverse health systems, thus our results should be extrapolated with caution in relation to other systems.

We hope that placing the consequences of respiratory infection in relation to the consequences of a hip fracture may provide a useful perspective for discussions of respiratory infection and its prevention with older populations. Acute respiratory infection is a critical event in the elderly. The population, especially the elderly but also their caregivers and clinical practitioners, should be more aware of this disease's risk and importance. Better recognition will improve the prevention of pneumonia and its deleterious consequences for the elderly over the short term (e.g. by increasing uptake of existing vaccines, such as for influenza and pneumococcus) and the long term (e.g. by increasing the prioritisation of pneumonia as an important biomedical research emphasis). Fighting pneumonia is essential to healthy ageing.

Antoine Guillon $^{1}$, Joseph P. Mizgerd ${ }^{2}$ and Leslie Grammatico-Guillon ${ }^{3}$

${ }^{1}$ CHRU de Tours, Service de médecine intensive réanimation, INSERM U1100, Centre d'Etude des Pathologies Respiratoires, Université de Tours, Tours, France. ${ }^{2}$ Pulmonary Center, Boston University School of Medicine, Boston, MA, USA. ${ }^{3} \mathrm{CHRU}$ de Tours, Unité d'épidémiologie des données cliniques régionales, Service d’information médicale, d'épidémiologie et d'économie de la santé, EA EES, Université de Tours, Tours, France.

Correspondence: Antoine Guillon, Service de Réanimation Polyvalente, CHRU Bretonneau, 2 Bd Tonnellé, F-37044 Tours Cedex 9, France. E-mail: antoine.guillon@univ-tours.fr

Received: 18 May 2020 | Accepted after revision: 28 July 2020

Provenance: Submitted article, peer reviewed.

Author contributions: A. Guillon and L. Grammatico-Guillon conceived and designed the study and were involved in 
drafting the manuscript. L. Grammatico-Guillon performed the data retrieval. A. Guillon and L. Grammatico-Guillon performed the statistical analysis. A. Guillon, L. Grammatico-Guillon and J.P. Mizgerd made substantial contributions to the conception of the study, interpretation of the data, and were involved in drafting the manuscript and made critical revisions of the discussion section. All authors read and approved the version to be published.

Conflict of interest: A. Guillon has nothing to disclose. J.P. Mizgerd reports US NIH grants related to pneumonia, including R35 HL135756, R01 AI115053, and R33 HL137081. L. Grammatico-Guillon has nothing to disclose.

\section{References}

1 Cillóniz C, Dominedò C, Pericàs JM, et al. Community-acquired pneumonia in critically ill very old patients: a growing problem. Eur Respir Rev 2020; 29: 190126.

2 Laporte L, Hermetet C, Jouan Y, et al. Ten-year trends in intensive care admissions for respiratory infections in the elderly. Ann Intensive Care 2018; 8: 84.

3 Fry AM, Shay DK, Holman RC, et al. Trends in hospitalizations for pneumonia among persons aged 65 years or older in the United States, 1988-2002. JAMA 2005; 294: 2712-2719.

4 International Federation on Ageing. A New View into Pneumonia Among Older Adults. ifa.ngo/publication/ health/new-view-pneumonia-among-older-adults/ Date last accessed: 26 September 2019.

5 Cillóniz C, Menéndez R, García-Vidal C, et al. Defining community-acquired pneumonia as a public health threat: arguments in favor from Spanish investigators. Med Sci (Basel) 2020; 8: 6.

6 Haentjens P, Magaziner J, Colón-Emeric CS, et al. Meta-analysis: excess mortality after hip fracture among older women and men. Ann Intern Med 2010; 152: 380-390.

7 Guillon A, Aymeric S, Gaudy-Graffin C, et al. Impact on the medical decision-making process of multiplex PCR assay for respiratory pathogens. Epidemiol Infect 2017; 145: 2766-2769.

8 Gilbert T, Neuburger J, Kraindler J, et al. Development and validation of a Hospital Frailty Risk Score focusing on older people in acute care settings using electronic hospital records: an observational study. Lancet 2018; 391: 1775-1782.

9 Jouan Y, Grammatico-Guillon L, Teixera N, et al. Healthcare trajectories before and after critical illness: population-based insight on diverse patients clusters. Ann Intensive Care 2019; 9: 126.

10 R Core Team. The R project for statistical computing. www.R-project.org Date last accessed: 18 May 2020.

11 Jouan Y, Grammatico-Guillon L, Espitalier F, et al. Long-term outcome of severe herpes simplex encephalitis: a population-based observational study. Crit Care 2015; 19: 345.

12 Grammatico-Guillon L, Baron S, Rosset P, et al. Surgical site infection after primary hip and knee arthroplasty: a cohort study using a hospital database. Infect Control Hosp Epidemiol 2015; 36: 1198-1207.

Copyright (C)ERS 2020

This article is open access and distributed under the terms of the Creative Commons Attribution Non-Commercial Licence 4.0 